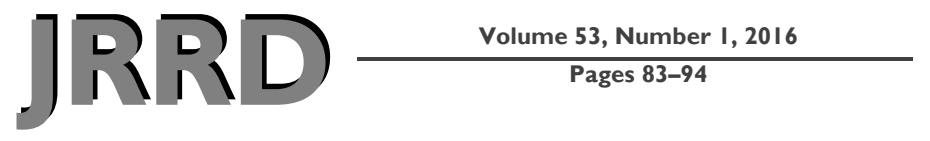

\title{
Sex differences between Veterans participating in interdisciplinary chronic pain rehabilitation
}

\author{
Jennifer L. Murphy, PhD; ${ }^{1-2 *}$ Kristin M. Phillips, PhD; ${ }^{1}$ Samantha Rafie, $\mathbf{P h D}^{\mathbf{1}}$ \\ ${ }^{1}$ James A. Haley Veterans' Hospital, Tampa, FL; ${ }^{2}$ University of South Florida, Tampa, FL
}

\begin{abstract}
The improved management of pain among the growing number of female Veterans receiving care through the Veterans Health Administration has been established as a priority, but studies suggest that females may respond differently to pain treatment. This study explored differences between female and male Veterans engaged in a Chronic Pain Rehabilitation Program and determined how female and male Veterans change following participation. Veterans $(N=324)$ in a $3 \mathrm{wk}$ inpatient program completed self-report measures at admission, discharge, and 3 mo follow-up. Participants were 21\% female $(n=67)$ and $79 \%$ male $(n=257)$. Compared with males, females were younger and less likely to be white or married/partnered. Females reported shorter pain duration and were more likely to have primary head or limb pain. At admission, fewer females were prescribed opioids than males and at lower doses. After opioid cessation in the program, however, there were no significant differences in use between the sexes at follow-up. Improvements in a range of domains were sustained at follow-up for both sexes, but females did not maintain gains in pain intensity or sleep while males reported more painrelated fear at discharge and follow-up. This study adds to the literature on sex-specific variations in chronic pain and implications for treatment.
\end{abstract}

Key words: chronic pain, females, gender, interdisciplinary treatment, multidisciplinary treatment, noncancer pain, opioids, pain rehabilitation, sex differences, treatment outcomes, Veterans, women.

\section{INTRODUCTION}

Chronic pain is prevalent among Veterans, and its treatment is a top priority for the Department of Veterans
Affairs (VA) [1]. Female Veterans, whose use of VA healthcare has almost doubled in the last decade, are the fastest growing subset of the Veteran population [2]. It is projected that women will comprise 10 percent of the population treated in the VA by 2018 and over 14 percent by 2033 [3]. Female Veterans are younger on average than men, with 42 percent between the ages of 18 and 44, while only 13 percent of males are under 45 [2]. Among Operation Iraqi Freedom (OIF), Operation Enduring Freedom (OEF), and Operation New Dawn (OND) Veterans, 51 percent of women are younger than 44 yr old [4]. In addition, female Veterans are more heterogeneous racially and ethnically, with 39 percent endorsing a minority background versus 23 percent of males [2].

\footnotetext{
Abbreviations: ANOVA = analysis of variance; $\mathrm{CBT}=$ cognitive behavioral therapy; CNP = chronic noncancer pain; CPRP = Chronic Pain Rehabilitation Program; CPS = chronic pain syndrome; CT = catastrophizing subscale; IMMPACT = Initiative on Methods, Measurement, and Pain Assessment in Clinical Trials; MED = morphine equivalent dose; NRS = numeric rating scale; OEF = Operation Enduring Freedom; OIF = Operation Iraqi Freedom; OND = Operation New Dawn; POQ-VA = Pain Outcomes Questionnaire-VA; SIS = Symptom Implausibility Scale; SPQ = Sleep Problems Questionnaire; VA = Department of Veterans Affairs.

*Address all correspondence to Jennifer L. Murphy, PhD; James A. Haley Veterans' Hospital, Pain Clinic G-38, 13000 Bruce B. Downs Blvd, Tampa, FL 33612; 813-9722000, ext 5239; fax: 813-631-6760.

Email: Jennifer.Murphy2@va.gov

http://dx.doi.org/10.1682/JRRD.2014.10.0250
} 
Data indicate that 57 percent of OIF/OEF/OND females have enrolled in and are using VA services [4]. Women most likely to enroll in VA care have greater psychosocial stressors such as low income, poor health status, and lack of social support [5].

Among the general population, chronic pain is more common in women than men [6]. Studies of experimental pain suggest that women exhibit heightened pain sensitivity [7], experience more intense and frequent pain [8], and report a broader range of pain locations [9]. There is less known about sex differences among the Veteran population specifically. In a large-scale study of OIF/OEF Veterans, the probability of having persistent pain did not differ between sexes, but women were more likely to experience moderate to severe pain [10]. Another study revealed that at years 1 through 7 postdeployment, women were more likely than men to have back, joint, and musculoskeletal pain, and the odds of having these conditions increased for women compared with men each year following deployment [11]. The most prevalent medical condition reflected in VA records for both sexes was musculoskeletal conditions at 55.9 percent for females and 48.5 percent for males [2].

The investigation of sex differences in nonpharmacological pain treatments is lacking and the data available are inconsistent. Jensen et al. found that women, but not men, undergoing cognitive behavioral therapy (CBT) with or without physical therapy exhibited improved health-related quality of life [12]. A study by Hansen et al. investigating various forms of back exercises found that men received greater benefit from conventional physiotherapy than women [13]. Krogstad et al. found that women had significantly more pain reduction than men 2 yr following conservative multimodal care for orofacial pain [14].

While the effectiveness of interdisciplinary pain rehabilitation is well established in the literature [15-17], there is a dearth of data comparing men and women in these settings. In a study of pain tolerance and pain program treatment outcomes between sexes, Edwards et al. found that females demonstrated greater improvement in pain-related disability while males showed more reduction in pain [18]. In addition, females with higher pain tolerances versus those with lower pain tolerances had greater improvements in pain, reduced pain-related interference with functioning, and more activity increases, while pain tolerance was not associated with positive treatment outcomes among males. A study of patients with fibromyalgia following an intensive, outpatient, interdisciplinary pain program showed a better outcome on some scales of the 36-Item Short Form Health Survey for males compared with females in a study by Hooten et al. [19]. Most similar to the current study, Keogh et al. compared 98 patients with complex pain syndromes who participated in a 3 to 4 wk group-based interdisciplinary pain management program at discharge and 3 mo followup [20]. Although both sexes showed similar gains at program completion, males maintained gains after 3 mo and females did not. Pieh et al. did not examine followup data but found that in a 5 wk daily outpatient pain program, females improved more than males despite there being no significant differences at program admission, including in areas such as pain duration, pain-related disability, and psychiatric comorbidities [21].

The growing number of female Veterans, many of whom will inevitably experience chronic pain, makes the acquisition of knowledge about their characteristics, conditions, and treatment of paramount importance to more effectively serve this population. While pain is a significant problem for both sexes in the VA, female Veterans may have additional risk factors for the development of chronic pain, such as higher rates of injury during initial training [22] as well as higher rates of depression [23] and military sexual trauma [24]. These factors and others may affect the optimal approach to pain care among females and the development of sex-specific pain programs.

To our knowledge, no previous studies have investigated sex differences in population characteristics or treatment outcomes within an interdisciplinary pain setting in the Veteran population. To address this issue, we compared multidomain treatment outcomes of female and male Veterans with chronic noncancer pain (CNP) who participated in a $3 \mathrm{wk}$ residential interdisciplinary chronic pain treatment program. Participants were assessed at three time points: admission, discharge, and 3 mo follow-up. The first aim was to explore differences in demographic and clinical variables between the sexes. The second aim was to determine whether there were differences between how females and males changed over time in pain outcomes. The findings in the literature in this area are limited and mixed; however, based on the most similar study in a civilian population [20], we hypothesized that both groups would demonstrate improvements from admission to discharge but that males would maintain more gains than females at the 3 mo follow-up. 


\section{METHODS}

\section{Participants and Procedures}

The current study is a retrospective data analysis of Veterans with CNP who were admitted to the inpatient Chronic Pain Rehabilitation Program (CPRP) at the James A. Haley Veterans' Hospital in Tampa, Florida, between August 2006 and April 2011. The CPRP is an intensive 3 wk residential, interdisciplinary chronic pain treatment program with a rehabilitation philosophy that seeks to assist those with CNP by teaching self-management skills that will improve quality of life and overall functioning. Patients who participate in the CPRP often have had little pain relief from various pharmacologic trials, interventional and surgical procedures, physical therapy, or complementary or alternative medicine approaches. They typically meet criteria for chronic pain syndrome (CPS) (International Classification of Diseases-9th RevisionClinical Modification code 338.4) [25], which is defined as chronic pain with significant psychosocial dysfunction. CPS is characterized by unsuccessful pain relief through conventional medical treatments; functional impairment in most domains of life; and negative emotional factors related to pain such as depression, anxiety, and irritability. The CPRP uses a biopsychosocial approach and targets the physical and emotional effects of pain with a focus on active treatment modalities including graduated physical therapy, aquatic therapy, daily paced walking, relaxation techniques, occupational therapy, recreational therapy, individual psychotherapy, educational groups, and family interventions as appropriate.

In addition, effective medication management is an important program goal and includes cessation of all opioids and centrally acting muscle relaxants. The use of other nonopioid analgesics are reviewed at admission and adjusted throughout treatment. Each participant's medication records were reviewed for the period in which they participated in the CPRP to confirm opioid use status at admission and to extract a taper dose. For those on opioids at admission, initial daily opioid dosing was calculated from the medication records based on the highest opioid taper dose dispensed in the first $3 \mathrm{~d}$ of the program. This dose was converted to a morphine equivalent dose (MED) for comparison purposes using established methods [26].

A cognitive-behavioral, biopsychosocial model serves as the basis of treatment. Treatment in the CPRP provides 6 to $8 \mathrm{~h}$ per day of supervised therapeutic pro- gramming during 15 consecutive workdays, coupled with an additional 2 to $3 \mathrm{~h}$ of daily independent, goal-directed assignments (e.g., walking and exercise program, relaxation techniques). Weekend treatment includes recreational and social activities as well as twice-daily exercise, walking, and relaxation sessions.

Prior to CPRP admission, those interested in participating in the program were evaluated for medical and psychiatric stability. Medical needs that may have precluded patients from full engagement and maximum benefit, thus excluding them from participation, included further evaluation by cardiology, neurosurgery, or pulmonology. Psychological barriers for participation included psychiatric hospitalization or illicit drug use within $90 \mathrm{~d}$ prior to screening. Those excluded from admission but interested in participating in the program were provided with treatment recommendations and rescreened at a future date.

\section{Measures}

Outcome measures reported in this study were administered at three time points. All participants initially completed measures within the first $2 \mathrm{~d}$ of admission to the CPRP. Participants were readministered and completed measures within $2 \mathrm{~d}$ of discharge from the program. Finally, they were mailed the questionnaires with return postage at 3 mo postdischarge and returned the packet via standard U.S. Postal Service. Questionnaires measured pain intensity and treatment outcomes across the major pain-related domains of functioning, catastrophizing, and sleep. Data regarding medications were retrospectively extracted from participants' electronic medical records.

Pain intensity was assessed using an 11-point pain numeric rating scale (NRS) to measure "usual” (average) pain over the last week. NRSs are reliable and valid methods for assessing pain intensity [27]. The NRS was anchored with the phrases "no pain" (0) and "worst pain imaginable" (10). The "usual pain" scale has been found to be one of the best measures of pain intensity when compared with alternatives such as "current pain" or "worst pain” [28].

Pain treatment outcomes were assessed using the Pain Outcomes Questionnaire-VA (POQ-VA) [29], which is a multidomain pain assessment instrument developed and validated specifically for Veterans. The POQ-VA assesses treatment outcomes across the major pain-related domains of functioning identified by the Rehabilitation 
Accreditation Commission (2002) as essential for comprehensive outcome measurement [29]. POQ-VA scales include interference with mobility, negative affect, vitality (i.e., strength and endurance), and pain-related fear (i.e., avoidance motivated by fear of pain or reinjury). The POQ-VA scales have been shown to have high internal reliability and good stability [30], strong generalizability, and good discriminant and concurrent validity, and they have demonstrated sensitivity to treatmentrelated change [31]. The POQ-VA also contains an experimental scale that was developed as a measure of highly improbable pain-related symptoms (the Symptom Implausibility Scale [SIS]). The SIS consists of 10 items describing a range of unusual pain symptoms or complaints.

Pain catastrophizing was assessed using the 6-item catastrophizing subscale (CT) from the revised 26-item Coping Strategies Questionnaire [32]. The CT has adequate internal consistency (0.72) and has been shown to negatively correlate with measures of activity [32]. Catastrophizing also has been positively correlated with depressive symptomatology [33-34], negative affectivity [35], exaggerated emotional response to aversive stimuli [36], and expectation of pain and psychological distress [37].

Sleep was assessed using the Sleep Problems Questionnaire (SPQ) [38]. The SPQ is a 4-item measure of the most typical symptoms of poor sleep in both healthy and distressed populations. Responses are based on the number of days during the week that each sleep symptom occurs, and these 0 to 7 item scores are summed for an overall sleep symptom measure. The scale has good internal consistency and validity [38].

\section{Data Analysis}

In preliminary analyses, the demographic and clinical characteristics of participants based on sex were compared using $t$-tests for continuous variables and chi-square tests for categorical variables. When categorical variables included more than two categories and the overall chisquare test was significant, subsequent Bonferronicorrected group comparisons were conducted for each category. To determine whether demographic and clinical variables for which there were significant differences between groups should be considered as potential control variables, the interaction with each of these variables with sex was evaluated for the pain variables at admission. Those variables that had significant interactions with sex would be retained as control variables.
To examine longitudinal changes in pain outcome variables across the three time points, repeated-measures analyses of variance (ANOVAs) were conducted using SPSS version 19 (IBM Corporation; Armonk, New York). Initial analysis consisted of a 2 (group: females, males) $\times 3$ (time: admission, discharge, follow-up) ANOVA for each outcome variable. The main effects of sex and time and interaction effects for sex $\times$ time were evaluated. Quadratic terms were included to determine whether there were nonlinear effects (e.g., plateaus or decrements in pain outcomes following improvement). Differences between males and females at each time point were evaluated using independent samples $t$-tests and within-group differences from admission to discharge and discharge to follow-up were evaluated using paired-samples $t$-tests. A $p$-value of 0.05 (two-tailed) was used for statistical significance. Effect sizes were calculated using Cohen's $d$. An effect size of 0.2 to 0.3 is considered small, 0.5 medium, and $\geq 0.8$ large [39]. The study was powered at 0.80 to detect an effect size of $f=$ 0.25 for the interaction between sex and time, assuming a per group sample size of 80 and alpha $=0.05$. The study was also powered at 0.80 to detect an effect size of $f=$ 0.14 for within-group change across the three measurement points.

\section{RESULTS}

\section{Preliminary Analyses}

Veterans $(N=324)$ completed self-report measures at admission, discharge, and 3 mo follow-up. Participants were 21 percent female $(n=67)$ and 79 percent male $(n=$ 257). Table 1 presents demographic and clinical characteristics of participants. Regarding demographic factors, there were several significant differences. Females were younger, with an average age of $47.19 \mathrm{yr}$, while males were $52.77 \mathrm{yr}$ on average $(t(322)=3.81, p<0.001)$. The majority of female participants in this study were unmarried and not cohabiting with a partner $(67 \%, n=45)$, compared with males who tended to be married or living with a partner $(63 \%, n=162)\left(\chi^{2}(1, N=324)=19.21\right.$, $p<0.001)$. Finally, females were less likely to be white $\left(\chi^{2}(2, N=324)=6.86, p=0.03\right)$, at 61 percent $(n=41)$ versus 76 percent $(n=195)$ of males. Regarding clinical factors, females had a shorter pain duration $(t(320)=$ 2.53, $p=0.01$ ) of $9.35 \mathrm{yr}$ on average versus males at $12.99 \mathrm{yr}$ on average. They were less likely to have a 
Table 1.

Differences between sexes on demographic and clinical factors. Data presented as mean \pm standard deviation or percentage.

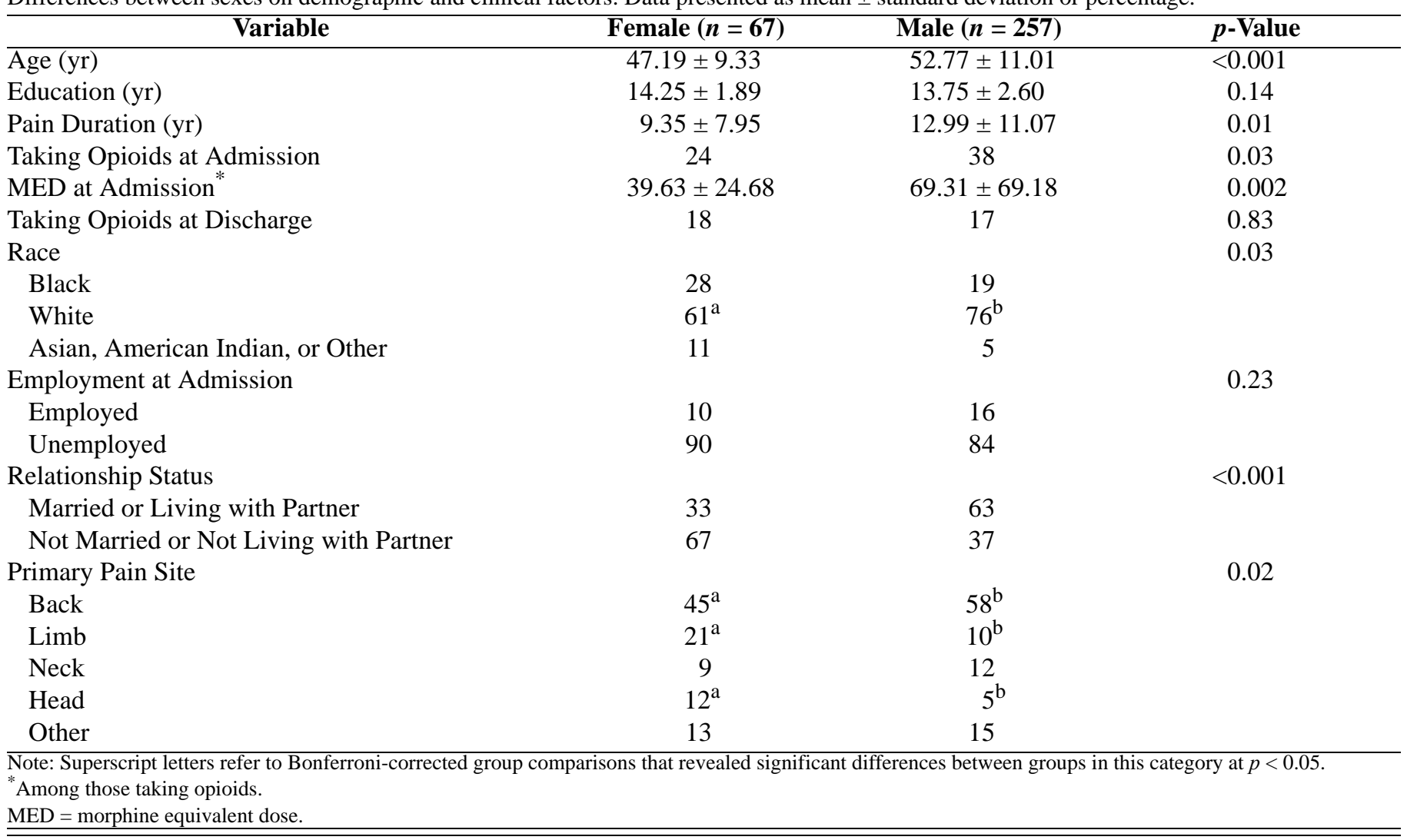

primary complaint of back pain (females: $45 \%, n=30$; males: $58 \%, n=149$ ), but more likely to report primary pain location in head (e.g., headaches) (females: 12\%, $n=8$; males: 5\%, $n=13)\left(\chi^{2}(4, N=324)=11.67, p=\right.$ 0.02 ) or the limb (e.g., leg, arm) (females: $21 \%, n=14$; males: $10 \%, n=26)$.

Regarding opioid use at admission, 35 percent of all participants were prescribed opioids; however, females were less likely to be prescribed opioids (24\%) than males $(38 \%)\left(\chi^{2}(1, N=324)=4.73, p=0.03\right)$. Among participants who were taking opioids, females had lower MEDs at admission compared with males $(t(62)=3.18, p=$ 0.002). Per CPRP standard of care, no participants were taking opioids at discharge. At follow-up, 17 percent of all participants reported opioid use; however, there were no differences in opioid use between females (18\%) and males $(17 \%)\left(\chi^{2}(1, N=312)=0.05, p=0.83\right)$.

Given the difference between sexes in the previously mentioned demographic and clinical factors, we evaluated whether any should be included as covariates by evaluating the effect of their interaction with sex on each outcome variable. Despite these differences, there were no significant interactions for sex $\times$ age, sex $\times$ marital status, sex $\times$ race, sex $\times$ pain duration, sex $\times$ primary pain location, or sex $\times$ opioid use for any outcome $(p>0.05)$; therefore, these variables were not included as covariates.

To evaluate differences between groups on pain outcomes at admission, we used $t$-tests. There were significant differences between groups on vitality, a scale measuring strength and endurance. Specifically, compared with males, females reported more pain-related interference in vitality $(t(322)=-2.10, p=0.04)$ (Table 2 ).

\section{Change Over Time in Pain Outcomes by Sex}

For pain intensity, there was a significant linear effect of time $(F(1,321)=16.70, p<0.001)$. However, there was no group $\times$ time interaction $(F(1,321)=0.12, p=$ $0.73)$, quadratic group $\times$ time interaction $(F(1,321)=$ $1.39, p=0.24)$, or main effect for group $(F(1,321)=0.02$, $p=0.88)$. There were no significant differences between sexes at any time point. Comparing time effects for each group separately indicated that both females and males 
JRRD, Volume 53, Number 1, 2016

Table 2.

Mean \pm standard deviation of outcome variables by group.

\begin{tabular}{|c|c|c|c|}
\hline Variable & Admission & Discharge & Follow-Up \\
\hline \multicolumn{4}{|l|}{ Average Pain Level } \\
\hline Female & $6.94 \pm 1.57$ & $6.07 \pm 1.93$ & $6.42 \pm 2.48$ \\
\hline Male & $6.88 \pm 1.73$ & $6.19 \pm 1.80$ & $6.26 \pm 2.06$ \\
\hline Female & $8.46 \pm 1.43$ & $7.97 \pm 1.80$ & $7.66 \pm 2.28$ \\
\hline Male & $8.50 \pm 1.29$ & $7.95 \pm 1.64$ & $7.89 \pm 1.85$ \\
\hline Male & $25.06 \pm 10.15$ & $21.72 \pm 10.55$ & $22.97 \pm 10.99$ \\
\hline \multicolumn{4}{|c|}{ Pain-Related Negative Effect } \\
\hline Female & $27.88 \pm 11.33$ & $21.84 \pm 11.98$ & $25.70 \pm 11.55$ \\
\hline Male & $28.05 \pm 11.34$ & $23.70 \pm 11.95$ & $27.55 \pm 12.56$ \\
\hline \multicolumn{4}{|c|}{ Pain Interference in Vitality } \\
\hline Female & $10.88 \pm 5.26$ & $7.25 \pm 4.27$ & $8.64 \pm 4.44$ \\
\hline Male & $11.77 \pm 4.55$ & $8.88 \pm 4.75$ & $10.60 \pm 4.78$ \\
\hline \multicolumn{4}{|l|}{ Implausible Symptoms } \\
\hline Female & $48.36 \pm 18.46$ & $32.04 \pm 20.02$ & $39.09 \pm 24.39$ \\
\hline Male & $47.19 \pm 20.75$ & $35.96 \pm 21.07$ & $42.24 \pm 24.11$ \\
\hline \multicolumn{4}{|l|}{ Sleep } \\
\hline Female & $21.48 \pm 5.76$ & $15.73 \pm 7.84$ & $20.05 \pm 8.65$ \\
\hline Male & $21.18 \pm 6.86$ & $17.80 \pm 7.99$ & $20.10 \pm 7.79$ \\
\hline \multicolumn{4}{|l|}{ Pain Catastrophizing } \\
\hline Female & $19.93 \pm 8.89$ & $12.33 \pm 8.37$ & $13.91 \pm 9.24$ \\
\hline Male & $19.03 \pm 9.56$ & $13.00 \pm 9.36$ & $16.22 \pm 10.31$ \\
\hline
\end{tabular}

reported improved pain from admission to discharge ( $p<$ $0.05)$. However, this reduction was only maintained at follow-up for males. That is, males reported improved pain from admission to follow-up $(t(255)=5.19, p<$ 0.001 ), whereas there was only a trend toward improvement in pain intensity for females from admission to follow-up $(t(66)=1.74, p=0.09)$. Effect sizes calculated from admission to discharge indicate small effects for males $(d=0.4)$ and medium effects for females $(d=0.5)$, and small effects for both at follow-up $(d=0.3)$.

For pain-related fear, a significant linear effect of time was found $(F(1,318)=26.17, p<0.001)$. There was also a significant main effect for group $(F(1,318)=8.25, p=$ 0.004) (Figure). Planned comparisons indicated that both groups demonstrated improvements from admission to discharge and from admission to follow-up $(p<0.05)$. However, comparing the sexes at each time point indicates that females and males reported equivalent levels of pain-related fear at admission $(t(322)=1.37, p=0.17)$,

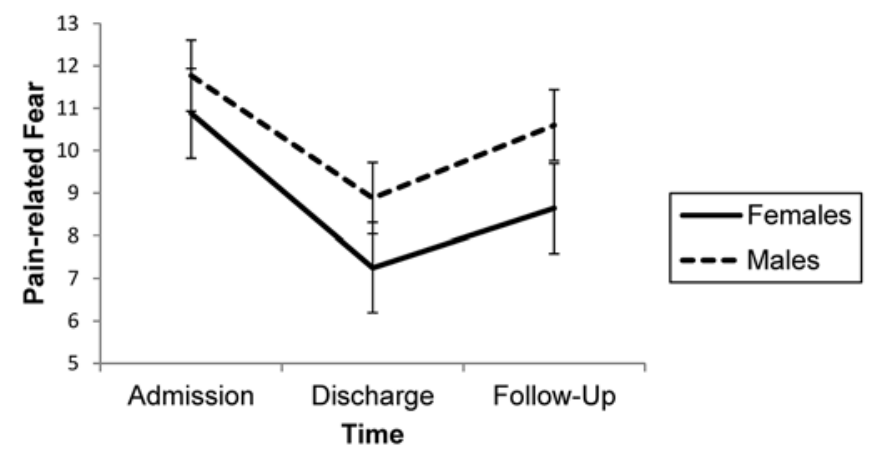

Figure.

Effect of sex on pain-related fear.

but males reported higher levels of pain-related fear than females at discharge $(t(322)=2.55, p=0.01)$ and followup $(t(318)=3.03, p=0.003)$. Effect sizes calculated from admission to discharge indicated medium effects for 
males $(d=0.6)$ and large effects for females $(d=0.8)$, which decreased at follow-up to small effects for males $(d=0.3)$ and medium effects for females $(d=0.5)$.

For mobility, a significant linear effect of time was found $(F(1,296)=17.08, p<0.001)$. However, there was no group $\times$ time interaction $(F(1,296)=0.87, p=0.35)$, quadratic group $\times$ time interaction $(F(1,296)=0.01, p=$ $0.94)$, or main effect for group $(F(1,296)=0.47, p=$ 0.49). There were no significant differences between sexes at any time point. Both females and males reported less pain-related interference with mobility from admission to discharge $(p<0.05)$, and both groups maintained this improvement at follow-up $(p<0.05)$, suggesting sex did not play a significant role in improvements in painrelated interference in mobility.

For pain-related negative affect, a significant quadratic effect of time was found $(F(1,317)=64.46, p<$ $0.001)$; however, the linear time effect was not significant $(p=0.08)$. There was no group $\times$ time interaction $(F(1,317)=1.14, p=0.29)$, quadratic group $\times$ time interaction $(F(1,317)=0.73, p=0.40)$, or main effect for group $(F(1,317)=0.86, p=0.36)$. There were no significant differences between sexes at any time point. Both females and males reported improved pain-related negative affect from admission to discharge $(p<0.05)$, but these effects were not maintained at follow-up. That is, males returned to their pretreatment levels of pain-related negative affect by follow-up $(t(252)=0.74, p=0.46)$ and females had only a trend toward improved affect from admission to follow-up $(t(66)=1.71, p=0.09)$.

For vitality, a significant linear effect of time was found $(F(1,313)=21.72, p<0.001)$. There was no group $\times$ time interaction $(F(1,313)=1.18, p=0.28)$, quadratic group $\times$ time interaction $(F(1,313)=0.03, p=0.87)$, or main effect for group $(F(1,313)=1.50, p=0.22)$. At admission, females reported higher levels of pain-related interference in vitality; however, there were no significant differences between the sexes at discharge or followup. Both groups demonstrated improvements from admission to discharge and from admission to follow-up $(p<0.05)$, suggesting sex did not play a significant role in improvements in pain-related interference in vitality.

For implausible symptoms, a significant linear effect of time was found $(F(1,280)=19.22, p<0.001)$. There was no group $\times$ time interaction $(F(1,280)=1.60, p=$ $0.21)$, quadratic group $\times$ time interaction $(F(1,280)=$ $0.00, p=0.97)$, or main effect for group $(F(1,280)=0.22$, $p=0.64)$. There were no significant differences between sexes at any time point. Both groups demonstrated improvements from admission to discharge and from admission to follow-up ( $p<0.05)$, suggesting sex did not play a significant role in improved reports of implausible symptoms.

For sleep, a significant linear effect of time was found $(F(1,303)=5.13, p=0.02)$. There was no group $\times$ time interaction $(F(1,303)=0.12, p=0.73)$, quadratic group $\times$ time interaction $(F(1,303)=1.72, p=0.19)$, or main effect for group $(F(1,303)=0.33, p=0.57)$. There were no significant differences between sexes at any time point. Comparing time effects for each group separately indicated that both groups demonstrated improvements from admission to discharge $(p<0.05)$. However, at follow-up this reduction was only maintained for males. That is, males reported improved overall sleep from admission to follow-up $(t(244)=2.21, p=0.03)$, whereas the change for females from admission to follow-up was not significant $(t(60)=1.38, p=0.17)$. Effect sizes calculated from admission to discharge indicated medium effects for males $(d=0.5)$ and large effects for females $(d=0.8)$, which decreased for both groups at follow-up to small effects ( $d=0.1$ and 0.2 , respectively).

For catastrophizing, a significant linear effect of time was found $(F(1,304)=23.41, p<0.001)$. There was no group $\times$ time interaction $(F(1,304)=2.45, p=0.12)$, quadratic group $\times$ time interaction $(F(1,304)=0.00, p=0.99)$, or main effect for group $(F(1,304)=0.75, p=0.39)$. There were no significant differences between the sexes at any time point. Both groups demonstrated improvements from admission to discharge and from admission to follow-up $(p<0.05)$, suggesting sex did not play a significant role in improvements in pain catastrophizing.

\section{DISCUSSION}

Consistent with previous findings, this study provides additional support regarding the benefits of interdisciplinary pain rehabilitation across a range of domains for both males and females. The results of this retrospective examination supported our hypothesis that while both males and females demonstrated significant improvements from admission to discharge, males maintained more gains than females at follow-up. The differential effects between sexes suggest potential considerations for future clinical care and investigation to maximize the benefits of interdisciplinary chronic pain treatment. 
Distinctions were found among sexes in several areas. Demographically, females were younger, less likely to be white, and less likely to be married or partnered. They had shorter pain durations, were less likely to have back pain, more likely to have head or limb pain, and less likely to be prescribed opioids. If taking opioids, they were on a lower daily MED. At admission, females reported more pain-related interference in vitality (i.e., strength and endurance), but there were no differences at discharge or follow-up. At discharge, both males and females reported significant improvements on all outcome variables; however, there were differences between males and females at the 3 mo follow-up.

While both sexes reported improved pain intensity from admission to discharge, females did not maintain pain reduction gains at 3 mo follow-up. This is similar to Keogh et al.'s finding, where females returned to baseline levels of pain intensity versus males [20]. While both sexes had statistically significant decreases in pain intensity at discharge, the effect sizes for females were medium, indicating a clinically significant change [29], whereas the effect size for males was small, meaning females actually made greater gains while in the program but were unable to maintain them. At 3 mo, effect sizes were small across sexes; however, it is important to note that the treatment modality is a tertiary-level inpatient pain rehabilitation program in which the population served represents those individuals who have the most chronic and treatment-refractory conditions. Because of this, the focus is on improving quality of life and function across domains despite the chronicity of the pain condition and any decrease in pain intensity is noteworthy.

In addition, while overall sleep improved during the course of program participation for both sexes, only males maintained gains in overall sleep at follow-up. The gains for both at discharge were statistically significant and the effect size for females was large, indicating a clinically significant change [29], whereas the effect size for males was medium. As with pain, females made greater gains while in the program but were unable to maintain them, and effects across sexes were small at 3 mo. While greater clinical significance at follow-up would have been preferred, the prevalence of sleep issues in the treated population, including sleep-disordered breathing conditions and medical comorbidities, make even small changes meaningful.

On the variable related to avoidance of activity due to a fear of increased pain and/or reinjury, females and males were equivalent at admission and both sexes demonstrated significant improvement over time; however, males reported higher levels of pain-related fear than females at both discharge and follow-up. Interestingly, in a study of sex and gender in the experience of pain, Ramírez-Maestre and Esteve found that fear-avoidance was associated with pain intensity in males but not females [40].

Both groups maintained improvements in mobility, vitality, endorsement of implausible symptoms, and catastrophizing. While some previous studies have found that females reported higher levels of catastrophizing, the results of the present study are consistent with RamírezMaestre and Esteve [40] and Unruh et al. [41] who did not find sex differences in this variable. Both females and males reported improved negative affect at program discharge, but the effects were not maintained for either group at follow-up.

This study highlights several areas for future research and clinical consideration. While the efficacy of interdisciplinary pain care has been well established in the literature, there is limited research examining sex differences in this treatment modality and available studies reflect inconsistent outcomes. Increased attention is warranted, and focusing on why and how specific aspects of treatment may affect the sexes differently would be particularly beneficial to enhance understanding. This would help to shed light on treatment decisions and inform whether programs should be altered based on the specific needs of females. Furthermore, future research should evaluate whether an intervention targeted to females can produce stronger effects and what aftercare treatment is most effective at maintaining those effects.

Across the literature regarding female Veterans with pain, the population is younger, and less likely to be white or married than their male peers. These consistent demographic findings have several potential implications. Females in the VA are more likely to be transitioning from the military and concomitantly contemplating choices about pursuing employment or additional education. Since they are more ethnically and racially diverse, it is essential to be aware of how their preferences and needs may differ. The fact that they are less likely to be married highlights the importance for independence both financially and in the home, as well as the potential implications of single parenting. When considering the treatment of females with pain or developing sex-specific programs, focusing on areas such as adjustment and transitional issues, 
vocational options, culturally sensitive diversity topics, gender roles (including parenting), and independent functioning should be emphasized. As is true of females in the general population, headaches were more common among females in this study. Because of this, it is also important to consider an enhanced role for the evaluation and treatment of headaches within pain rehabilitation programs.

Examining differences in VA and Department of Defense prescribing practices for females versus males also warrants further investigation. While lower dosing is likely, at least in part because of average weight differences between the sexes, in this study, females were also less likely to be prescribed opioids at all. This finding conflicts with evidence from the public sector that females are actually more likely to be prescribed opioids. The reasons for the opioid use patterns in this sample are unclear, although there are several possibilities. Since chronic opioid therapy is typically not indicated for headache pain, which was more prevalent among females, that may account for some of the difference. Females may have received higher dosages of other analgesics such as muscle relaxants, though we do not know because medications other than opioids were not assessed at admission. It is also possible that females were undertreated or undermedicated compared with males [42]. Additionally, the literature indicates that females are more likely to be referred to mental health specialists when presenting for pain complaints [41].

Perhaps the most important issue that this study raises is determining the optimal follow-up needs for females as well as males following intensive rehabilitation. Effect sizes ranged from small to large at discharge and tended to decrease at follow-up. This trend indicates that all patients would likely benefit from additional maintenance treatment following program discharge to sustain effects. Programmatically, clinical responses in the CPRP have been to schedule an earlier postdischarge visit or contact (i.e., at 1 mo vs $3 \mathrm{mo}$ ) to evaluate implementation and make any needed interventions as soon as possible. Aftercare options have also been expanded to include several stepped groups to expand duration. However, although effect sizes were generally small at followup, suggesting limited clinical significance, the modest improvements should be balanced against the fact that those in the program had longstanding CPS and the vast majority of Veterans who tapered off of opioid analgesics during treatment did not return to use.
While females did as well as or better than males during treatment, their self-reported pain intensity did not remain significantly improved 3 mo after discharge, which was consistent with previous findings [20]. They also did not retain sleep gains, and neither group maintained improvements in negative affect at follow-up. For females, since they made greater gains in several areas during treatment but did not maintain them, enhanced aftercare should be considered strongly in any sex-specific pain programs. Identifying specific information about why regression for both sexes may have occurred is important in understanding how to prevent the return to baseline functioning in these areas in the future. Clarification regarding the obstacles that may have prevented the implementation of acquired self-management strategies would be useful in the development of an enhanced plan of care prior to discharge and may improve long-term outcomes. Furthermore, since the cohort that receives comprehensive tertiary-level care is likely to have greater psychiatric needs than the general chronic pain population regardless of sex, more intensive mental health support following treatment may be indicated, as the lack of maintenance regarding affective gains suggests.

Finally, the consideration of issues more prevalent among female Veterans should be examined in future research and treatment approaches. For example, since military sexual trauma is reported at screening among one in four female Veterans [43], consideration of options that address both physical pain and trauma are indicated to optimize the efficiency and effectiveness of treatment. Likewise, tailoring treatment to accommodate conditions seen most commonly in females such as headaches, fibromyalgia, and pelvic pain may better serve the needs of this special population. Furthermore, some evidence suggests that using a different therapeutic approach with females such as Acceptance and Commitment Therapy may be more beneficial than a CBT-guided curriculum focused on controlling or changing unwanted thoughts and feelings.

The current study has several limitations that should be acknowledged. First, this was a retrospective design that did not include a control group; thus, no definitive conclusions can be made regarding the intervention. Second, only pain location and negative affect, as reflected on the POQ-VA, were known for program participants. Specific medical and psychiatric diagnostic information was not available and likely would have been helpful in gaining a fuller understanding of the role of comorbidities. 
Third, the generalizability of this sample to the general population may be restricted because it was only 21 percent female and all participants had a military history. However, given that females constitute slightly less than 10 percent of the cohort registered for VA care, the higher number of females in this sample likely accurately reflects the prevalence of chronic pain among female Veterans. In addition, having only one point of follow-up at 3 mo was a limitation because we were unable to examine sustained differences over a longer period such as 6 or 12 mo. Finally, while this was not a clinical trial, the use of Initiative on Methods, Measurement, and Pain Assessment in Clinical Trials (IMMPACT)-recommended measures might have helped in making conclusions more standardized and generalizable. The selected measures were chosen for use in a clinical program due to their applicability to the population; specifically, the POQ-VA is the only pain outcomes measure that was developed and validated for Veterans. Furthermore, IMMPACT core domains such as pain intensity and various key functional areas such as negative affect and mobility are reflected in the POQ-VA.

\section{CONCLUSIONS}

The current findings add to a growing body of research suggesting that sex differences may exist in the determinants of pain treatment outcomes. Given the increase in the number of female Veterans entering the VA system, these data are likely to address gaps in our current knowledge of female Veterans' experiences and needs in pain management and have clinically relevant implications for better serving female Veterans with chronic pain. Additional examination of the differences that may exist between sexes and their implications for effective pain management should continue to be explored.

\section{ACKNOWLEDGMENTS}

\author{
Author Contributions: \\ Study concept and design: J. L. Murphy, K. M. Phillips. \\ Acquisition of data: J. L. Murphy, K. M. Phillips. \\ Statistical analysis and interpretation of data: K. M. Phillips, S. Rafie. \\ Drafting of manuscript: J. L. Murphy, K. M. Phillips, S. Rafie. \\ Critical revision of manuscript for important intellectual content: \\ J. L. Murphy, K. M. Phillips, S. Rafie. \\ Final approval of manuscript: J. L. Murphy, K. M. Phillips, S. Rafie.
}

Financial Disclosures: The authors have declared that no competing interests exist.

Funding/Support: This material was unfunded at the time of manuscript preparation.

Additional Contributions: Dr. Rafie is now with the Bay Area Pain and Wellness Center, Los Gatos, California.

Institutional Review: Approval for the data used in this study was obtained from the James A. Haley Veterans' Hospital Research and Development Review Committee and the University of South Florida Institutional Review Board. Informed consent was not obtained because this is a retrospective study.

Participant Follow-Up: The authors do not plan to inform participants of the publication of this study.

Disclaimer: All opinions expressed herein are those of the authors and do not in any way represent those of the VA, Department of Defense, U.S. Government, or any of its agencies.

\section{REFERENCES}

1. Veterans Health Administration. Pain management. VHA directive 2009-053 [Internet]. Washington (DC): Department of Veterans Affairs; 2009 Oct 28. Available from: http://www.va.gov/painmanagement/docs/ vha09paindirective.pdf

2. Frayne SM, Phibbs CS, Saechao F, Maisel NC, Friedman SA, Finlay A, Berg E, Balasubramanian V, Dally SK, Ananth L, Romodan Y, Lee J, Iqbal S, Hayes PM, Zephyrin L, Whitehead A, Torgal A, Katon JG, Haskell S. Sourcebook: Women veterans in the Veterans Health Administration. Vol. 3: Sociodemographics, utilization, costs of care, and health profile [Internet]. Palo Alto (CA): Women's Health Evaluation Initiative, Women's Health Services, Veterans Health Administration, Department of Veterans Affairs; [2014 Feb]. Available from: http://www.womenshealth.va.gov/WOMENSHEALTH/ docs/Sourcebook_Vol_3_FINAL.pdf

3. Yano EM, Hayes P, Wright S, Schnurr PP, Lipson L, BeanMayberry B, Washington DL. Integration of women veterans into VA quality improvement research efforts: What researchers need to know. J Gen Intern Med. 2010;25(1 Suppl 1):56-61. [PMID:20077153] http://dx.doi.org/10.1007/s11606-009-1116-4

4. Department of Veterans Affairs. Fact sheet: Women veterans health care [Internet]. Washington (DC): Office of Public Affairs, Media Relations; 2012 [updated 2013 Apr 23]. Available from: http://www.womenshealth.va.gov/womenshealth/ docs/women veterans healthcare factsheet.pdf

5. Washington DL, Yano EM, Simon B, Sun S. To use or not to use. What influences why women veterans choose VA health care. J Gen Intern Med. 2006;21(Suppl 3):S11-18. [PMID:16637939] http://dx.doi.org/10.1111/j.1525-1497.2006.00369.x 
6. Gureje O, Von Korff M, Simon GE, Gater R. Persistent pain and well-being: A World Health Organization study in primary care. JAMA. 1998;280(2):147-51.

[PMID:9669787]

http://dx.doi.org/10.1001/jama.280.2.147

7. Fillingim RB, King CD, Ribeiro-Dasilva MC, RahimWilliams B, Riley JL 3rd. Sex, gender, and pain: A review of recent clinical and experimental findings. J Pain. 2009; 10(5):447-85. [PMID:19411059]

http://dx.doi.org/10.1016/j.jpain.2008.12.001

8. Unruh AM. Gender variations in clinical pain experience. Pain. 1996;65(2-3):123-67. [PMID:8826503] http://dx.doi.org/10.1016/0304-3959(95)00214-6

9. Dao TT, LeResche L. Gender differences in pain. J Orofac Pain. 2000;14(3):169-84, discussion 184-95. [PMID:11203754]

10. Haskell SG, Brandt CA, Krebs EE, Skanderson M, Kerns RD, Goulet JL. Pain among Veterans of Operations Enduring Freedom and Iraqi Freedom: Do women and men differ? Pain Med. 2009;10(7):1167-73. [PMID:19818028] http://dx.doi.org/10.1111/j.1526-4637.2009.00714.x

11. Haskell SG, Ning Y, Krebs E, Goulet J, Mattocks K, Kerns R, Brandt C. Prevalence of painful musculoskeletal conditions in female and male veterans in 7 years after return from deployment in Operation Enduring Freedom/Operation Iraqi Freedom. Clin J Pain. 2012;28(2):163-67.

[PMID:21677563]

http://dx.doi.org/10.1097/AJP.0b013e318223d951

12. Jensen IB, Bergström G, Ljungquist T, Bodin L, Nygren AL. A randomized controlled component analysis of a behavioral medicine rehabilitation program for chronic spinal pain: Are the effects dependent on gender? Pain. 2001; 91(1-2):65-78. [PMID:11240079] http://dx.doi.org/10.1016/S0304-3959(00)00420-6

13. Hansen FR, Bendix T, Skov P, Jensen CV, Kristensen JH, Krohn L, Schioeler H. Intensive, dynamic back-muscle exercises, conventional physiotherapy, or placebo-control treatment of low-back pain. A randomized, observer-blind trial. Spine (Phila Pa 1976). 1993;18(1):98-108.

[PMID:8434332]

http://dx.doi.org/10.1097/00007632-199301000-00015

14. Krogstad BS, Jokstad A, Dahl BL, Vassend O. The reporting of pain, somatic complaints, and anxiety in a group of patients with TMD before and 2 years after treatment: Sex differences. J Orofac Pain. 1996;10(3):263-69.

[PMID:9161231]

15. Flor H, Fydrich T, Turk DC. Efficacy of multidisciplinary pain treatment centers: A meta-analytic review. Pain. 1992; 49(2):221-30. [PMID:1535122] http://dx.doi.org/10.1016/0304-3959(92)90145-2

16. Gatchel RJ, Okifuji A. Evidence-based scientific data documenting the treatment and cost-effectiveness of compre- hensive pain programs for chronic nonmalignant pain. J Pain. 2006;7(11):779-93. [PMID:17074616]

http://dx.doi.org/10.1016/j.jpain.2006.08.005

17. Scascighini L, Toma V, Dober-Spielmann S, Sprott H. Multidisciplinary treatment for chronic pain: A systematic review of interventions and outcomes. Rheumatology (Oxford). 2008;47(5):670-78. [PMID:18375406]

http://dx.doi.org/10.1093/rheumatology/ken021

18. Edwards RR, Doleys DM, Lowery D, Fillingim RB. Pain tolerance as a predictor of outcome following multidisciplinary treatment for chronic pain: Differential effects as a function of sex. Pain. 2003;106(3):419-26.

[PMID:14659525]

http://dx.doi.org/10.1016/j.pain.2003.09.004

19. Hooten WM, Townsend CO, Decker PA. Gender differences among patients with fibromyalgia undergoing multidisciplinary pain rehabilitation. Pain Med. 2007;8(8):624-32. [PMID:18028040] http://dx.doi.org/10.1111/j.1526-4637.2006.00202.x

20. Keogh E, McCracken LM, Eccleston C. Do men and women differ in their response to interdisciplinary chronic pain management? Pain. 2005;114(1-2):37-46.

[PMID:15733629]

http://dx.doi.org/10.1016/j.pain.2004.12.009

21. Pieh C, Altmeppen J, Neumeier S, Loew T, Angerer M, Lahmann C. Gender differences in outcomes of a multimodal pain management program. Pain. 2012;153(1):197-202. [PMID:22100358] http://dx.doi.org/10.1016/j.pain.2011.10.016

22. Bell NS, Mangione TW, Hemenway D, Amoroso PJ, Jones BH. High injury rates among female Army trainees: A function of gender? Am J Prev Med. 2000;18(3 Suppl): 141-46. [PMID:10736550] http://dx.doi.org/10.1016/S0749-3797(99)00173-7

23. Maguen S, Ren L, Bosch JO, Marmar CR, Seal KH. Gender differences in mental health diagnoses among Iraq and Afghanistan veterans enrolled in Veterans Affairs health care. Am J Public Health. 2010;100(12):2450-56. [PMID:20966380] http://dx.doi.org/10.2105/AJPH.2009.166165

24. Kimerling R, Street AE, Pavao J, Smith MW, Cronkite RC, Holmes TH, Frayne SM. Military-related sexual trauma among Veterans Health Administration patients returning from Afghanistan and Iraq. Am J Public Health. 2010; 100(8):1409-12. [PMID:20558808] http://dx.doi.org/10.2105/AJPH.2009.171793

25. Buck CJ, Netter FH. 2012 ICD-9-CM for hospitals: Volumes 1, 2 \& 3. St. Louis (MO): Elsevier/Saunders, American Medical Association; 2012.

26. Gammaitoni AR, Fine P, Alvarez N, McPherson ML, Bergmark S. Clinical application of opioid equianalgesic data. 
Clin J Pain. 2003;19(5):286-97. [PMID:12966254]

http://dx.doi.org/10.1097/00002508-200309000-00002

27. Jensen MP, Karoly P. Self-report scales and procedures for assessing pain in adults. In: Turk DC, Melzack R, editors. Handbook of pain assessment. 2nd ed. New York (NY): Guilford Press; 2001. p. 15-34.

28. Fordyce WE. Behavioral methods for chronic pain and illness. St. Louis (MO): Mosby; 1976.

29. Clark ME, Gironda RJ, Young RW. Development and validation of the Pain Outcomes Questionnaire-VA. J Rehabil Res Dev. 2003;40(5):381-95. [PMID:15080223] http://dx.doi.org/10.1682/JRRD.2003.09.0381

30. Gironda RJ, Azzarello L, Clark ME. Pscyhometrics: Reliability of the National Pain Data Bank. Am J Pain Mgmt. 2002;12(1):24-30.

31. Clark ME, Gironda RJ. Assessment: Concurrent validity of the National Pain Data Bank: Preliminary results. Am J Pain Mgmt. 2000;10(1):25-33.

32. Riley JL 3rd, Robinson ME. CSQ: Five factors or fiction? Clin J Pain. 1997;13(2):156-62. [PMID:9186023] http://dx.doi.org/10.1097/00002508-199706000-00010

33. Geisser ME, Robinson ME, Keefe FJ, Weiner ML. Catastrophizing, depression and the sensory, affective and evaluative aspects of chronic pain. Pain. 1994;59(1):79-83. [PMID:7854806] http://dx.doi.org/10.1016/0304-3959(94)90050-7

34. Sullivan MJ, D’Eon JL. Relation between catastrophizing and depression in chronic pain patients. J Abnorm Psychol. 1990;99(3):260-63. [PMID:2145334] http://dx.doi.org/10.1037/0021-843X.99.3.260

35. Hirsh AT, George SZ, Riley JL 3rd, Robinson ME. An evaluation of the measurement of pain catastrophizing by the coping strategies questionnaire. Eur J Pain. 2007; 11(1):75-81. [PMID:16545973] http://dx.doi.org/10.1016/j.ejpain.2005.12.010

36. Sullivan MJ, Bishop SR, Pivik J. The Pain Catastrophizing Scale: Development and validation. Psychol Assess. 1995; 7:524-32. http://dx.doi.org/10.1037/1040-3590.7.4.524

37. Sullivan MJ, Rodgers WM, Kirsch I. Catastrophizing, depression and expectancies for pain and emotional dis- tress. Pain. 2001;91(1-2):147-54. [PMID:11240087]

http://dx.doi.org/10.1016/S0304-3959(00)00430-9

38. Jenkins CD, Stanton BA, Niemcryk SJ, Rose RM. A scale for the estimation of sleep problems in clinical research. J Clin Epidemiol. 1988;41(4):313-21. [PMID:3351539] http://dx.doi.org/10.1016/0895-4356(88)90138-2

39. Cohen J. Statistical power analysis for the behavioral sciences. 2nd ed. Hillsdale (NJ): L. Erlbaum Associates; 1988.

40. Ramírez-Maestre C, Esteve R. The role of sex/gender in the experience of pain: Resilience, fear, and acceptance as central variables in the adjustment of men and women with chronic pain. J Pain. 2014;15(6):608-18.e1.

[PMID:24632112]

http://dx.doi.org/10.1016/j.jpain.2014.02.006

41. Unruh AM, Ritchie J, Merskey H. Does gender affect appraisal of pain and pain coping strategies? Clin J Pain. 1999;15(1):31-40. [PMID:10206565] http://dx.doi.org/10.1097/00002508-199903000-00006

42. Institute of Medicine. Relieving pain in America: A blueprint for transforming prevention, care, education, and research. Washington (DC): National Academies Press; 2011.

43. MST Support Team. Military sexual trauma [Internet]. Washington (DC): Department of Veterans Affairs; 2015 May. Available from: http://www.mentalhealth.va.gov/ docs/mst_general_factsheet.pdf

Submitted for publication October 22, 2014. Accepted in revised form March 9, 2015.

This article and any supplementary material should be cited as follows:

Murphy JL, Phillips KM, Rafie S. Sex differences between Veterans participating in interdisciplinary chronic pain rehabilitation. J Rehabil Res Dev. 2016;53(1):83-94. http://dx.doi.org/10.1682/JRRD.2014.10.0250

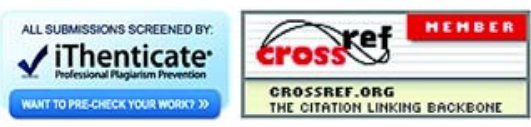

